ISAHP 2001,Berne, Switzerland, August 2-4,2001

\title{
THE CATEGORICAL DATA STATISTICAL ANALYSIS FOR THE PAIRWISE COMPARISONS IN RATIO SCALE
}

\author{
Indrani Basak \\ Penn State at Altoona \\ 3000 Ivyside Park \\ Altoona, PA 16601 \\ USA \\ li8b@psu.edu
}

Keywords: Asymptotic Distribution; Iterative Procedure;Logistic Distribution; Logit Model; Maximum Likelihood Estimator;Hypothesis Testing.

Summary: Pairwise comparison methods involving ratio scale models are considered. The pairwise comparison values sometimes are cardinal representations of ordered categorical variables. This model arises in Saaty's scaling method of priorities which is referred to as Analytic Hierarchy Process (AHP). In this article, we develop a logit model for the ordered response variable in the AHP. Relevant estimation and hypothesistesting procedures are addressed in this perspective. 
